Proyecciones

Vol. 18, No 2, pp. 195-205, December 1999

Universidad Católica del Norte

Antofagasta - Chile

\title{
ON (*)-IDEALS AND POSITIVE IMPLICATIVE IDEALS IN BCI-ALGEBRAS
}

\author{
HAMZA A. S. ABUJABAL \\ King Abdul Aziz University, Saudi Arabia \\ and \\ JIE MENG \\ Northwest University Xian, China
}

\begin{abstract}
In the present paper, we given some equivalent conditions of $\left({ }^{*}\right)$ ideals and positive implicative ideals in BCI-algebras and we clarify the relation of the two classes of ideals. Also, we obtain further properties of these ideals. Finally, we discuss a quotient algebra of a $B C I$-algebra by a closed (*)-ideal and a quotient algebra by positive implicative ideal.
\end{abstract}

KEY WORDS AND PHRASES : BCI-algebra, closed ideal, $\left(^{*}\right)$-ideal, positive implicative ideal, quotient algebra.

1991 AMS Subject classification : 03G25, 06F35. 
The study of ideals forms an important part of the theory of BCIalgebra. Since $K$. Iséki [6] generalized the notion of ideals in BCK-algebras to BCI-algebras, several classes of ideals in BCI-algebras have occurred, for instance, closed ideals [2], strong ideals [1], $p$-ideals [16], positive implicative ideals [3], and so on. In [4] and [9] closed ideals, strong ideals, and $p$-ideals were further investigated. In particular, it is shown that in a BCI-algebra the notion of strong ideals and closed $(*)$-ideals coincide. As a continuation of [4] and [9], we now, will deeply study further properties of (*)-ideals and positive implicative ideals and clarify the relation of the two classes of ideals.

Let $X$ be a nonempty set. Let ${ }^{*}$ be a binary operation on $X$ and 0 is a constant of $X$. An algebra $\langle X ; *, 0\rangle$ of type $(2,0)$ is said to be a BCI-algebra if for all $x, y, z \in X$,

(I) $((x * y) *(x * z)) *(z * y)=0$,

(II) $(x *(x * y)) * y=0$,

(III) $x * x=0$,

(IV) $x * y=0$ and $y * x=0$ imply $x=y$

A binary relation $\leq$ on $X$ can be defined by putting $x \leq y$ if and only if $x * y=0$. Then $\langle X ; \leq\rangle$ is a partially ordered set with a minmal element 0 .

In any BCI-algebra $X$ the following properties hold :

(1) $(x * y) * z=(x * z) * y$,

(2) $x * 0=x$,

(3) $(x * z) *(y * z) \leq x * y$

(4) $x \leq y$ implies $x * z \leq y * z$ and $z * y \leq z * x$.

A BCI-algebra $X$ with the condition $0 \leq x$ for all $x \in X$ is called a BCK-algebra.

A nonempty subset $I$ of a BCI-algebra $X$ is called an ideal of $X$ if

(i) $0 \in I$,

(ii) $x \in I$ whenever $x * y \in I$ and $y \in I$.

Every ideal $I$ of $X$ satisfies

(iii) $x \leq y$ and $y \in I$ imply $x \in I$.

An ideal $I$ of a BCI-algebra $X$ is said to be closed if $0 * x \in I$ for all $x \in I$. An ideal $I$ of a BCI-algebra $X$ is closed if and only if $I$ is a subalgebra of $X$. Every ideal of a BCK-algebra is always closed. An element $a$ in a BCI-algebra $X$ is said to be an atom if for all $x \in X, x * a=0$ implies $x=a$. Let $L(X)$ be the set of all atoms of a BCI-algebra $X$. For any $a \in L(X)$, the set $\{x \in X \mid a \leq x\}$ is called the branch of a BCI-algebra $X$ and denoted by $V(a)$. The branch $V(0)$ is the BCK-part of $X$, which 
is denoted by $B(X)=\{x \in X \mid 0 \leq x\}$. For all $x$ in a BCI-algebra $X$, $a_{x}=0 *(0 * x) \in L(X)$. In the sequel, we will use the following properties:

(5) $L(X)$ is a subalgebra of $X$,

(6) $L(X)=\{0 *(0 * x) \mid x \in X\}=\{0 * x \mid x \in X\}$,

(7) If $x, y \in X$, then $x$ and $y$ belong to the same branch if and only if $x * y \in B(X)$ and so $x * a_{x} \in B(X)$.

A BCI-algebra $X$ is said to be $p$-semisimple if $B(X)=\{0\}$ or equivalently, $L(X)=X$.

The above concepts and results can be found in [1], [7] and [12]. Throughout this paper, $X$ will mean a BCI-algebra unless mentioned otherwise.

Before starting to discuss $(*)$-ideals and positive implicative ideals, we give an elementary property of $\mathrm{BCI}$-algebras.

Theorem 1 : Let $X$ be a BCI-algebra. Then for all $x \in X$ and $y \in B(X), x * y \leq x$.

Proof : Since $y \in B(X)$ implies $0 * y=0$, we have $(x * y) * x=$ $(x * x) * y=0 * x=0$, that is $x * y \leq x$. $\diamond$

E. H. Roh, Y. B. Jun and S. M. Wei [13] introduced the notion of (*)-ideals in BCI-algebras and obtain some of its properties.

Definition $1^{[13]}:$ If an ideal $I$ of a BCI-algebra $X$ satisfies the condition

(iv) $x \in I$ and $a \in X-I$ imply $x * a \in I$, then $I$ is called a (*)-ideal of $X$.

Obviously, each ideal of a BCK-algebra is always a (*)-ideal, but for a proper BCI-algebra, $\{0\}$ is not a $(*)$-ideal.

Theorem $2:$ If $I$ is an ideal of a BCI-algebra $X$, and $L(X) \subseteq I$, then $I$ is a $(*)$-ideal of $X$.

Proof : Assume that $x \in I$ and $y \in X-I$. Since $y * a_{y} \in B(X)$, by Theorem 1 , we have $x *\left(y * a_{y}\right) \leq x$, and so $x *\left(y * a_{y}\right) \in I$. Since

$$
\begin{aligned}
\left((x * y) * a_{y}\right) *\left(x *\left(y * a_{y}\right)\right) & =\left(\left(x *\left(x *\left(y * a_{y}\right)\right)\right) * y\right) * a_{y} \\
& \leq\left(\left(y * a_{y}\right) * y\right) * a_{y} \\
& =\left(0 * a_{y}\right) * a_{y} \in L(X),
\end{aligned}
$$


and $L(X) \subseteq I$, we have $\left((x * y) * a_{y}\right) *\left(x *\left(x * a_{y}\right)\right) \in I$. Combining $x *\left(y * a_{y}\right) \in I$ and using (ii), we have $(x * y) * a_{y} \in I$. Since $a_{y} \in L(X) \subseteq I$, it follows that $x * a \in I$. Therefore, $I$ is a $(*)$-ideal of $X$. $\diamond$

The converse of Theorem 2 need not be hold, as is shown in the following example.

Example $1^{[9]}$ : Let $X=\left\{2^{n} \mid n= \pm 1, \pm 2, \ldots\right\}$, and let $\div$ be the usual division. Then $\langle X ; \div, 1\rangle$ is a $p$-semisimple BCI-algebra and $I=\left\{1,2,2^{2}, \ldots\right\}$ is an ideal of $X$. Observe that for all natural numbers $m$ and $n$, we have $2^{-n} \in X-I, 1 \div 2^{-n} \in I$ and $2^{m} \div 2^{-n}=2^{m+n} \in I$. Hence mIis a $(*)$-ideal of $X$. But $L(X) \nsubseteq I$ as $L(X)=X$.

One easily sees that $I$ is not closed, because $1 \div 2=\frac{1}{2} \notin I$.

It is natural to ask whether or not for closed ideals the converse of Theorem 2 holds. The answer is positive.

Theorem 3 : Let $I$ be a closed $(*)$-ideal of a BCI-algebra. Then $L(X) \subseteq I$.

Proof : If $L(X) \nsubseteq I$, then there is $a \in L(X)-I$, that is, $a \in L(X)$ and $a \notin I$. But $a \in L(X)$ implies $a=0 *(0 * a)$. By $(i v), a \notin I$ implies $0 * a \in I$. Furthermore, $a=0 *(0 * a) \in I$, because $I$ is closed. Therefore, we have a contradiction. Hence $L(X) \subseteq I$. $\diamond$

By Theorem 2 and Theorem 3, we have

Corollary 4 : If $I$ is a closed ideal of a BCI-algebra $X$, then $I$ is a (*)-ideal if and only if $L(X) \subseteq I$.

Definition $2^{[11]}$ : Let $\mathrm{N}$ be the set of all natural numbers. Let $X$ be a BCI-algebra. For $x \in X$, we define $x^{n}$ by $x^{1}=x, x^{n+1}=x *\left(0 * x^{n}\right)$. If there is $n \in \mathrm{N}$ such that $x^{n} \in B(X)$, then $x$ is called finite periodic and $|x|=\min \left\{n \in \mathbf{N} \mid x^{n} \in B(X)\right\}$ is the period of $x$. The set $P(X)=$ $\{x \in X|| x \mid<\infty\}$ is called the periodic part of $X$. If $X=P(X)$, then $X$ is said to be periodic. 
Proposition 5 [11, Theorem 11] : Let $X$ be a periodic BCI-algebra. Then each ideal of $X$ is closed.

Combing Corollary 4 and Proposition 5, we get

Theorem 6 : If $X$ is a periodic BCl-algebra, and $I$ is an ideal of $X$, then $I$ is a $(*)$-ideal of $X$ if and only if $L(X) \subseteq I$.

Since a finite BCI-algebra is periodic (see [11, Theorem 8], we obtain

Corollary 7 : An ideal $I$ of a finite BCl-algebra is a $(*)$-ideal if and only if $l(X) \subseteq I$.

Now, we give simpler characterizations of $(*)$-ideals and closed $(*)$ ideals.

Theorem 8 : Suppose $I$ is an ideal of a BCl-algebra $X$. Then $I$ is a (*)-ideal of $X$ if and only if $a \in X-I$ implies $0 * a \in I$.

Proof $(\Longrightarrow)$ : Trivial.

$(\Longleftarrow)$ : Suppose an ideal $I$ satisfies that $a \in X-I$ implies $0 * a \in I$. If $x \in I$ and $a \in X-I$, then $(x * a) * x=(x * x) * a=0 * a \in I$. By (ii), $x * a \in I$. Therefore, $I$ is a $(*)$-ideal. $\diamond$

Theorem 9 : An ideal $I$ of a BCl-algebra $X$ is a closed (*)-ideal if and only if $0 * x \in I$ for all $x \in X$.

Proof : Let $I$ be a closed (*)-ideal and $x \in X$. If $x \in X-I$, then $0 * x \in I$, because $I$ is a $(*)$-ideal. If $x \in I$, then $0 * x \in I$, because $I$ is a closed ideal. Hence $0 * x I$, for all $x \in X$.

Conversely, suppose $0 * x \in I$ for all $x \in X$. In other words, $L(X) \subseteq I$ by (6). It follows from the definition of closed ideals that $I$ is closed. Then, applying Corollary 4 , we get that $I$ is a $(*)$-ideal. $\diamond$.

Theorem 10 : A nonempty subset $I$ of a BCI-algebra $X$ is a closed $(*)$-ideal of $X$ if and only if $(i) 0 \in I$, and $(v)$ for all $x, y, z \in X, x * y \in I$ and $y \in I$ imply $x * z \in I$. 
Proof: Suppose that $I$ satisfies $(i)$ and $(v)$. Assume that $z=0$ in $(v)$. Then $I$ satisfies $x * y \in I$ and $y \in I$ imply $x \in I$. Hence $I$ is an ideal of $X$. Let $x=y=0$ in $(v)$. Then $0 * z \in I$ for all $z \in X$. By Theorem 9 , is a closed $(*)$-ideal of $X$.

Conversely, let $I$ be a closed (*)-ideal of $X$. If $x * y \in I$ and $y \in I$, then by closeness of $I$, we have $x * z \in I$ whenever $z \in I$. Thus, for all $x, y, z \in Z, x * y \in I$ and $y \in I$ imply $x * z \in I . \diamond$

For a subset $A$ of $X$, let $(A]$ resp. $\left.(A]_{*}\right)$ denotes the least ideal (resp. least closed (*)-ideal) containing $A$ in $X$.

Theorem 11 : Let $A$ be a subset of a BCI-algebra $X$. Then $(A]_{*}=$ $(A \cup L(X)]$.

Proof : It follows directly from Theorem 9 and (6).

Next, we discuss positive implicative ideals in BCI-algebras and their relation with $(*)$-ideals. The notion of positive implicative ideals in BCKalgebras was introduced by K.Iséki in [5] and generalized to BCI-algebras by C. S. Hoo in [3].

Definition $3^{[5]}$ : A nonempty subset $I$ of a BCI-algebras $X$ is called a positive implicative ideal of $X$ if it is satisfies $(i) 0 \in I$ and $(v i)(x * y) *$ $z \in I$ imply $x * z \in I$.

Any positive implicative ideal must be an ideal, but the converse need not be hold.

Definition $4^{[14]}$ : A BCI-algebra $X$ is quasi-associative if for $x, y, z \in$ $X,(x * y) * z \leq x *(y * z)$.

Proposition $12^{[14]}$ : A BCI-algebra $X$ is quasi-associative if and only if for all $x \in X, 0 * x \leq x$, or equivalently $(0 * x) * x=0$.

For a nonempty subset $A$ of a BCI-algebra $X$, and a fixed element $a$ of $X$ denote $A_{a}=\{x \in X \mid x * a \in A\}$. 
Theorem 13 : Let $X$ be a quasi-associative BCI-algebra and let $A$ be a positive implicative ideal of $X$. Then for any fixed $a \in X, A_{a}$ is the least ideal containing $A$ and $a$.

Proof : Since $X$ is quasi-associative, $(0 * a) * a=0 \in A$. Combining $a * a=0 \in A$ any by $(v i)$, we have $0 * a \in A$, that is, $0 \in A_{a}$. Also, let $x * y \in A_{a}$ and $y \in A_{a}$. Then $(x * y) * a \in A$ and $y * a \in A$. By (vi), $x * a \in A$ and so $x \in A_{a}$. Therefore, $A_{a}$ is an ideal of $X$.

Since $X$ is quasi-associative, for all $x \in X$, we have $(x * a) * a \leq x *$ $(a * a)=x * 0=x \in A$ and so $(x * a) * a \in A$ by (iii). Observe that $a * a=0 \in A$. Hence $x * a \in A$. Namely, $x \in A_{a}$. Thus $A \subseteq A_{a}$. Clearly, $a \in A_{a}$.

Suppose that $I$ is any ideal containing $A$ and $a$. If $x \in A_{a}$, then $x * a \in$ $A \subseteq I$, and so $x * a \in I$. It follows from $a \in I$ that $x \in I$. Hence $A_{a} \subseteq I$. This means that $A_{a}$ is the least ideal containing $A$ and $a$. $\diamond$

Theorem 14: Let $A$ be an ideal of a BCI-algebra $X$. If for all $a \in X$, $A_{a}$ is an ideal of $X$, then $A$ is a positive implicative ideal of $X$.

Proof : Let $x, y, z \in X,(x * y) * z \in A$ and $y * z \in A$. Then $x * y \in A_{z}$ and $y \in A_{z}$. Since $A_{z}$ is an ideal of $X$, by (ii) $x \in A_{z}$ and so $x * z \in A$. Hence $A$ is a positive implicative ideal of $X$. $\diamond$

By Theorem 13 and Theorem 15, we have

Corollary 15 : Let $X$ be a quasi-associative BCI-algebra and $A$ be an ideal of $X$. Then $A$ is positive implicative if and only if for any $a \in X, A_{a}$ is an ideal of $X$.

Çthe following result is a generalization of [8, Theorem 4].

Theorem 16 : If $I$ is an ideal of a BCI-algebra $X$, then the following are equivalent : (8) $I$ is positive implicative,

(9) $(x * y) * y \in I$ implies $x * y \in I$, for all $x, y \in X$,

(10) $(x * y) * z \in I$ implies $(x * z) *(y * z) \in I$ for all $x, y, z \in I$. 
Theorem 17 : Suppose $X$ is a quasi-associative BCI-algebra and $A$ is a positive implicative ideal of $X$. Then $A$ is a closed (*)-ideal of $X$.

Proof : For all $a \in X, A_{a}$ is an ideal of $X$ by Theorem 13. Since $0 \in A_{a}$, it follows that $0 * a \in A$. By Theorem 9 , we know that $A$ is a closed (*)-ideal of $X$. $\diamond$

Example 2 : Let $X=\{0,1,2,3,4,5\}$ and the ${ }^{*}$ table is given by

$\begin{array}{lllllll}* & 0 & 1 & 2 & 3 & 4 & 5 \\ 0 & 0 & 0 & 0 & 3 & 3 & 3 \\ 1 & 1 & 0 & 0 & 3 & 3 & 3 \\ 2 & 2 & 2 & 0 & 5 & 5 & 3 \\ 3 & 3 & 3 & 3 & 0 & 0 & 0 \\ 4 & 4 & 3 & 3 & 1 & 0 & 0 \\ 5 & 5 & 5 & 3 & 2 & 2 & 0\end{array}$

Then $\langle X: *, 0\rangle$ is a quasi-associative BCI-algebra and $I=\{0,3\}$ is closed (t)-ideal of $X$, but $I$ is not positive implicative as $(4 * 3) * 3=$ $1 * 3=3 \in I$ and $4 * 3=1 \notin I$. Hence the converse of Theorem 17 does not hold.

Corollary $18^{[15]}$ : Let $I$ be a positive implicative ideal of a quasiassociative BCI-algebra $X$. Then $L(X) \subseteq I$.

Proof : It follows from Theorem 3 and Theorem 17. $\diamond$

By quotient algebras, we can characterize $(*)$-ideals. Let $I$ be an ideal of a BCI-algebra $X$. Define a binary relation $\sim$ on $x$ by $x \sim y$ if and only if $x * y \in I$. Then $\sim$ is a congruence relation on $X$. Let $C_{x}=\{y \in X \mid y \sim x\}$ and $X / I=\left\{C_{x} \mid x \in X\right\}$. Define $C_{x} * C_{y}=C_{x * y}$. Then $C_{0}$ is the greatest closed ideal contained in $I$, and $\left\langle X / I ; *, C_{0}\right\rangle$ is a BCI-algebra, called the quotient algebra of $X$ by $I$ (see [7]).

Theorem 19 : Let $I$ be a closed ideal of a BCI-algebra $X$. Then $I$ is a $(*)$-ideal if and only if $\left\langle X / I ; *, C_{0}\right\rangle$ is a BCK-algebra. 
Proof $(\Longrightarrow)$ : Suppose $I$ is a closed $(*)$-ideal of $X$. By Theorem 9, $0 *$ $x \in I$ for all $x \in X$, and so $C_{0} * C_{x}=C_{0 * x}=I=C_{0}$ for all $x \in X$. Hence $\left\langle X / I ; *, C_{0}\right\rangle$ is a BCK-algebra.

$(\Longleftarrow)$ : If $\left\langle X / I ; *, C_{0}\right\rangle$ is a BCK-algebra, then for all $x \in X, C_{0} * C_{x}=$ $C_{0}$. Thus $C_{0 * x}=I$. Hence $0 * x \in I$. By Theorem $9, I$ is a closed (*)-ideal of $X . \diamond$

Corollary 20 : Let $X$ be a quasi-associative BCI-algebra, and let $I$ be a positive implicative ideal of $X$. Then $\left\langle X / I ; *, C_{0}\right\rangle$ is a positive implicative BCK-algebra.

Proof : By Theorem 17 and Theorem 19, it suffices to prove that $\left\langle X / I ; *, C_{0}\right\rangle$ is positive implicative. So, we assume that $\left(C_{x} * C_{y}\right) * C_{y} \in$ $\left\{C_{0}\right\}$. Hence $C_{(x * y) * y}=I$, and so $(x * y) * y \in I$. By (9), we have $x * y \in I$. By (9), we have $x * y \in I$. Thus $C_{x} * C_{y}=C_{x * y}=C_{0} \in\left\{C_{0}\right\}$. This is to 6 say that the zero ideal $\left\{C_{0}\right\}$ is positive implicative in BCK-algebra $X / I$. By $[8$, Corollary 7$],\left\langle X / I ; *, C_{0}\right\rangle$ is a positive implicative BCK-algebra. $\diamond$

\section{References}

1. S. A. Bhatti, Close-ness and open-ness of ideals in BCI-algebras, Math. Japan, 36, pp. 915-921, (1991).

2. C. S. Hoo, Closed ideals and p-semisimple BCI-algebras, Math. Japon, 35, pp. 1103-1112, (1990).

3. C. S. Hoo, Filters and ideals in BCI-algebras, Math. Japon, 36, pp. 987-997, (1991).

4. S. M. Hong, Y. B. Jun and J. Meng, On strong ideals and p-ideals in BCI-algebras, submitted.

5. K. Iséki, On ideals in BCK-algebras, Math. Seminar Notes, 3, pp. $1-12,(1975)$.

6. K. Iséki, On BCI-algebras, Math. Seminar Notes, 8, pp. 125-130, (1980). 
7. T. D. Lei and C. C. Xi, p-radical in BCI-algebras, Math. Japon, 30, pp. $511-517,(1985)$.

8. J. Meng, On ideals in BCK-algebras, Math. Japon, 40, pp. 143-154, (1994).

9. J. Meng and H. A. S. Abujabal, On closed ideals in BCI-algebras, submitted.

10. J. Meng and Y. B. Jun, BCK-algebras, Kyung Moon Sa Co., Seoul, Korea, (1994).

11. J. Meng and S. M. Wei, Periodic BCI-algebras and closed ideals in BCI-algebras, Math. Japon, 38, pp. 571-575, (1993).

12. J. Meng and X. L. Xin, Characterizations of atoms in BCI-algebras, Math. Japon, 37, pp. 359-361, (1992).

13. E. H. Roh, Y. B. Jun and S. M. Wei, Some ideals in BCI-algebras, Math. Japon, to appear.

14. C. C. Xi, On a class of BCI-algebras, Math. Japon, 35, pp. 13-17, (1990).

15. Q. Zhang, BCI-algebras with weak units, Math. Japon, 36, pp. 11631166, (1991).

16. X. H. Zhang, On p-ideals of a BCI-algebras, Math. Japon, submitted.

Received : March, 1998 


\section{Hamza A. S. Abujabal}

Department of Mathematics

Faculty of Science

King Abdul Aziz University

P. O. Box 31464

Jeddah 21497

Saudi Arabia

and

\section{Jie Meng}

Department of Mathematics

Northwest University Xian

710069 P. R. China

China 